\title{
NONSINGULAR SECTIONS TO EUCLIDEAN BUNDLES $\left({ }^{1}\right)$
}

\author{
BY \\ KENNETH C. MILLETT( ${ }^{(1)}$
}

1. Introduction. Let $H(n+k ; n)$ denote the topological group of homeomorphisms of $R^{n+k}$ which leave 0 and the $n$-dimensional hyperplane, $R^{n} \times 0$, invariant. A Euclidean bundle, or an $\left(R^{n+k}, R^{n}\right)$-bundle, over a space, $X$, is defined to be a fiber bundle with fiber, $R^{n+k}$, and group, $H(n+k ; n)$. The total space of a Euclidean bundle contains a subspace which has the (induced) structure of an $\left(R^{n}, 0\right)$-bundle over $X$. Thus $\left(\xi^{n+k}, \xi^{n}\right)$ will denote an $\left(R^{n+k}, R^{n}\right)$-bundle with total space, $\left(E_{\xi^{n+k}}, E_{\xi^{n}}\right)$, and projection, $p$.

If $n=0$, we take $R^{0}$ to be 0 and also use 0 to denote $\xi^{0}$. The trivial $\left(R^{k}, 0\right)$-bundle is $\left(\varepsilon^{k}, 0\right)$. Since every Euclidean bundle contains 0 and $p: E_{0} \rightarrow X$ is always a homeomorphism, there is an inverse, $\sigma_{0}$, which is called the zero section after the following definition. Let $\left(\xi^{n+k}, \xi^{n}\right)$ be a Euclidean bundle over $X$. A section is a map $\sigma: X \rightarrow E_{\xi^{n+k}}$ such that $p \sigma=1_{X}$, where $1_{X}$ denotes the identity map on $X$. A section is said to be nonsingular if $\sigma(X) \subset E_{\xi^{n+k}} \backslash E_{\xi^{n}}$, where $A \backslash B$ denotes those points of $A$ not in $B$.

Just as in the theory of vector bundles, it is possible to define the Whitney sum of Euclidean bundles. We write

$$
\left(\xi^{n+k}, \xi^{n}\right) \oplus\left(\eta^{m+l}, \eta^{m}\right)=\left(\xi^{n+k} \oplus \eta^{m+l}, \xi^{n} \oplus \eta^{m}\right) .
$$

If a Euclidean bundle, $\left(\xi^{n+k}, \xi^{n}\right)$, is equivalent to $\left(\xi^{n} \oplus \xi^{k}, \xi^{n}\right)=\left(\xi^{n}, \xi^{n}\right) \oplus\left(\xi^{k}, 0\right)$ we say that the bundle splits. A splitting need not exist. Indeed, the RourkeSanderson embedding, [15] and [13], gives a Euclidean bundle which splits only in a very weak sense, i.e. as a spherical fibration [12].

The purpose of this work is to investigate the relationship between the existence of a nonzero section and a splitting of the Euclidean bundle. For example, if a vector bundle has a nonzero section it splits off an $\varepsilon^{1}$. Thus, one asks if a nonzero section to an $\left(R^{k}, 0\right)$-bundle implies that it splits off an $\varepsilon^{1}$. Browder [2], using results of Hirsch [6] and Mazur, has shown that an $\left(R^{k}, 0\right)$-bundle with a nonzero section splits off an $\varepsilon^{1}$ if and only if it contains a closed disk bundle. (By Kister [9], this means that the $\left(R^{k}, 0\right)$-bundle must be equivalent to the interior of a $\left(D^{k}, 0\right)$ bundle, where $D^{k}$ is the closed $k$-disk, whose (topological) group is the group of

Received by the editors January 24, 1969.

(1) This research was partially supported by the National Science Foundation under Grant GP-5860 and contains part of the author's doctoral dissertation written under the direction of Professor Edward Fadell. 
homeomorphisms of $D^{k}$ leaving 0 fixed.) Browder then proves that there is an $\left(R^{k}, 0\right)$-bundle over a finite simplicial complex which has a nonzero section, but which does not split off an $\varepsilon^{1}$. Consequently, it is of interest to know why there is no splitting and, furthermore, if there is a homotopy splitting.

Browder's result implies that one of the following statements is false.

(A) A Euclidean bundle, $\left(\xi^{k}, 0\right)$, over a finite simplicial complex with a nonzero section is equivalent to $\left(\xi^{k}, \varepsilon^{1}\right)$.

(B) A Euclidean bundle, $\left(\xi^{k+1}, \varepsilon^{1}\right)$, over a finite simplicial complex splits.

Motivated by the suspicion that (B) is false in the topological category and desiring to determine conditions under which $(\mathrm{A})$ is true, we are led to consider the following, more general, question. Is a Euclidean bundle, $\left(\xi^{n+k+1}, \xi^{n}\right)$, having a nonsingular section equivalent to $\left(\xi^{n+k+1}, \xi^{n} \oplus \varepsilon^{1}\right)$ ?

In the stable range this question may be answered by using the recent results of Kirby and Siebenmann [8], or Lashof and Rothenberg [10], relating the topological category to the piecewise linear category and then applying the results of Haefliger and Wall [5]. It is below this stable range that our results are of interest and provide a new application of piecewise linear techniques to topological bundle problems. This is done by generalizing the techniques developed by A. V. Černavskii [4].

In the following we shall assume that $X$ is a topological space which has the homotopy type of a countable CW-complex of dimension $m$.

THEOREM 1. Suppose that $\left(\xi^{n+k+1}, \xi^{n}\right)$ is a Euclidean bundle over $X$ such that $k \geqq 3$ and $m+n \leqq 2 k-3$. Then, if $\sigma$ is a nonsingular section to the bundle, the Euclidean bundle is equivalent to $\left(\xi^{n+k+1}, \xi^{n} \oplus \varepsilon^{1}\right)$, where $E_{\varepsilon^{1}}$ contains $\sigma(X)$. If $m+n \leqq 2 k-4$, $\varepsilon^{1}$ is unique.

This theorem is proved by "reducing the group" of the bundle. Thus, let $e_{j}$, $1 \leqq j \leqq k$, denote the point in $R^{k}$ whose only nonzero coordinate is a 1 in the $j$ th position. The topological group of homeomorphisms of $R^{n+k}$ which are the identity on $R^{n} \times 0$ will be denoted by $G(n+k ; n)$. Let $G_{1}(n+k ; n)$ denote the subgroup of $G(n+k ; n)$ consisting of those homeomorphisms leaving $e_{n+1}$ fixed. In $\S 2$ the following theorem is used to give the necessary reduction.

THeOREM 2. If $k \geqq 4$ and $m+n \leqq 2 k-6$, then

$$
\pi_{m}\left(G_{1}(n+k ; n), G(n+k ; n+1)\right)=0 .
$$

Theorem 2 is then reduced to a geometric bundle problem which can be considered in a manner analogous to that used by Cernavskir. As a final comment on Theorem 2, we note that the dimensional restrictions are necessitated by the use of general position and engulfing arguments in Lemma 4.11 and Proposition 5.1 .

A corollary to Theorem 1 gives a partial verification of statement (A). Unfortunately, it does not seem to have sufficient strength to prove that (B) is false. 
Corollary 3. Suppose that $\left(\xi^{k+1}, 0\right)$ is a Euclidean bundle over $X$, with $k \geqq 3$ and $m \leqq 2 k-3$. If the bundle has a nonzero section, it is equivalent to $\left(\xi^{k+1}, \varepsilon^{1}\right)$, where $\sigma(X) \subset E_{\varepsilon}$. If $m \leqq 2 k-4, \varepsilon^{1}$ is unique.

2. Reduction to a geometric bundle problem. In this section we shall, assuming Theorem 2, give proofs of Theorem 1 and its corollaries. Theorem 2 is then reduced to a bundle problem which is accessible by geometric methods.

Proof of Theorem 1. By Whitehead [17], we may assume that $X$ is a countable, locally finite, $m$-dimensional polyhedron. Let $G$ be any topological group and $B G$ the associated classifying space for $G$-bundles [14]. Given a $G$-bundle, $\xi$, let $f_{\xi}$ denote its classifying map.

It is easy to show that an $\left(R^{n+k+1}, R^{n}\right)$-bundle, $\left(\xi^{n+k+1}, \xi^{n}\right)$, has a nonsingular section if and only if

$$
f_{\left(\xi^{n+k+1}, \xi^{n}\right)}: X \rightarrow B H(n+k+1 ; n)
$$

factors (homotopically) through $B H_{1}(n+k+1 ; n)$, where $H_{1}(n+k+1 ; n)$ is the subgroup of $H(n+k+1 ; n)$ leaving $e_{n+1}$ fixed.

We note that $H_{1}(n+k+1 ; n)$ is (group) homeomorphic to

$$
G(n ; 0) \times G_{1}(n+k+1 ; n)
$$

and that the theorem will be proved if we can show that, in the appropriate dimensional range, any map

$$
g: X \rightarrow B H_{1}(n+k+1 ; n)
$$

can be (homotopically) factored through $B G(n ; 0) \times G(n+k+1 ; n+1)$. The obstruction to doing this is in $\pi_{m}\left(B H_{1}(n+k+1 ; n), B G(n ; 0) \times G(n+k+1 ; n+1)\right)$ which is isomorphic to $\pi_{m-1}\left(G_{1}(n+k+1 ; n), G(n+k+1 ; n+1)\right)$. If $m+n \leqq 2 k-3$, and $k \geqq 3$, this group is trivial by Theorem 2 . Hence existence has been proven. The uniqueness statement follows similarly.

Corollary 3 follows from Theorem 1 by taking $n=0$.

With the above, it is only necessary to prove Theorem 2 . Let $\alpha$ be an element of $\pi_{m}\left(G_{1}(n+k ; n), G(n+k ; n+1)\right)$ and let

$$
G:\left(\Delta^{m}, \partial \Delta^{m}\right) \rightarrow\left(G_{1}(n+k ; n), G(n+k ; n+1)\right)
$$

be a map representing $\alpha$. Corresponding to $G$, via the adjoint homeomorphism, there is a bundle map, also denoted by $G$, such that the diagram

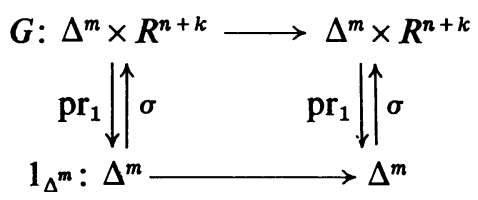

is commutative, where $\sigma(x)=\left(x, e_{n+1}\right)$. Thus, to show that $\alpha$ is zero, it suffices to prove that a representative $G$, can be written as the composition of bundle maps 
$G_{1}, G_{2}$ and $G_{3}$, where $G_{3}$ represents a map into $G(n+k ; n+1)$ and $G_{1}$ and $G_{2}$ are homotopic to bundle maps in $G(n+k ; n+1)$ through bundle maps in

$$
\left(G_{1}(n+k ; n), G(n+k ; n+1)\right) .
$$

We note that we may assume, without loss of generality, that $G$ is radially constant in a neighborhood of $\partial \Delta^{m}$. Thus it is sufficient to prove the following theorem.

THEOREM 2.1. If $m+n \leqq 2 k-6,4 \leqq k, s<1$, and

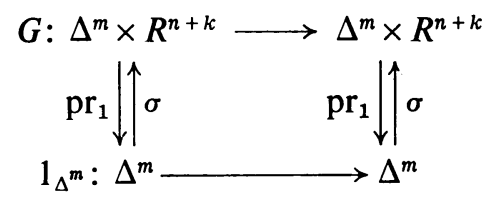

is a bundle map such that

(i) the diagram commutes,

(ii) $G$ is the identity on

$$
\operatorname{cl}\left(\Delta^{m} \backslash \Delta_{s}^{m}\right) \times R^{n+1} \cup \Delta^{m} \times R^{n}
$$

then $G=G_{1} G_{2} G_{3}$ such that, for some $t<1$,

(i) $G_{i}$ is $f$-isotopic to the identity through bundle maps having the same properties as $G$, with $s$ replaced by $t, s<t<1, i=1,2$,

(ii) $G_{3}$ represents a map of $\Delta^{m}$ into $G(n+k ; n+1)$.

Proof of Theorem 2.1. By Proposition 5.2, $G$ can be written as the composition $G_{1} G_{2} G_{3}$ such that $G_{1}$ satisfies the hypothesis of Lemma $4.13, G_{2}$ satisfies the hypothesis of Lemma 4.12 , and $G_{3}$ satisfies condition (iii). Since, by the Lemmas 4.12 and $4.13, G_{1}$ satisfies (i) and $G_{2}$ satisfies (ii), Theorem 2.1 is proven.

For the most part, the remainder of this paper is devoted to giving a proof of Proposition 5.2. The basic definitions and conventions necessary for the development of the theory are given in §3. In addition to Lemmas 4.12 and 4.13 , a series of lemmas are given in $\S 4$ which are used to give a proof of Proposition 5.2 in $\S 5$. The proofs of many of the results will be omitted as they are direct extensions of published proofs. The proof of Proposition 5.2 is based upon a result of A. V. Černavskiǐ [4]. Since the author has been unable to significantly simplify Černavskiǐ's argument we shall give an outline of the proof and include the necessary modifications.

3. Definitions and conventions. Consider a coordinate system for $R^{n+k}$ given by $x_{1}, \ldots, x_{n+k}$ and consider $R^{l} \subset R^{n+k}$ as $R^{l}=\left\{\left(x_{1}, \ldots, x_{n+k}\right) \mid x_{l+1}=\cdots=x_{n+k}=0\right\}$. Let

$$
\begin{aligned}
R_{n}^{+} & =\left\{\left(x_{1}, \ldots, x_{n+k}\right) \mid x_{n+1} \geqq 0\right\}, \\
R_{n}^{-} & =\left\{\left(x_{1}, \ldots, x_{n+k}\right) \mid x_{n+1} \leqq 0\right\}, \\
R_{+}^{n+1} & =R^{n+1} \cap R_{n}^{+}, \\
R_{-}^{n+1} & =R^{n+1} \cap R_{n}^{-}, \\
D_{t}^{l} & =\left\{x \in R^{l} \mid\|x\| \leqq t\right\} .
\end{aligned}
$$


Define $\Delta_{t}^{l}$ inductively by

$$
\Delta_{t}^{0}=\Delta^{0}=\{0\}, \quad \Delta_{t}^{l}=\Delta_{t}^{l-1} *\left\{\left(t e_{l}\right),\left(-t e_{l}\right)\right\},
$$

where * denotes the geometric join in $R^{l}$. Let

$$
\Delta_{t+}^{n+1}=\Delta_{t}^{n+1} \cap R_{+}^{n+1}, \quad \Delta_{t-}^{n+1}=\Delta_{t}^{n+1} \cap R_{-}^{n+1}
$$

and identify $\Delta_{1}^{l}$ with $\Delta^{l}$ via the obvious piecewise linear homeomorphism.

At every point of $R^{n}, x$, consider the family of two dimensional planes in $R^{n+k}$, $\left\{P_{\alpha, x}\right\}$ coordinatized by the line in $R^{n+1}$, through $x$, parallel to the $x_{n+1}$ axis as abscissa and a line orthogonal to $R^{n+1}$ as ordinate. We note that the various families are mutually disjoint and that $P_{\alpha, x}$ and $P_{\beta, x}$ have only their abscissa in common. In each $P_{\alpha, x}$ consider all possible semicircles, $\left\{C_{\beta, \alpha, x}\right\}$, with centers at $x$ and having their diameters lying in the abscissa. Note that through each point of $R^{n+k} \mid R^{n+1}$ there passes exactly one semicircle $C_{\beta, \alpha, x}$ and exactly one plane $P_{\alpha, x}$.

We now define a family of surfaces which will play a crucial role in the argument. These surfaces, called horns and denoted by $R_{t}$, consist of the points in $R^{n+k}$ which satisfy the equation

$$
x_{n+1}=t\left(x_{n+2}^{2}+\cdots+x_{n+k}^{2}\right)^{1 / 2}, \quad-\infty<t<+\infty .
$$

Let $R_{-\infty}=R_{-}^{n+1}$ and $R_{+\infty}=R_{+}^{n+1}$.

Each horn, $R_{t},-\infty<t<+\infty$, intersects each plane, $P_{\alpha, x}$, in two rays originating at $x$ lying on both sides of the abscissa and forming, with it, equal angles of arccotangent $(t)$. As $t$ varies between $-\infty$ and $+\infty$, each point on the horn describes a semicircle which belongs to a family $\left\{C_{\beta, \alpha, x}\right\}$. For each horn we may thus define the positive and negative side (relative to the $x_{n+1}$ axis) and for each pair of horns, the region between them. These regions will be denoted by $Q_{t}^{+}, Q_{t}^{-}$and $Q_{\left(t_{1}, t_{2}\right)}$ respectively.

A set $X$ is said to be tangential to $R_{t}$ if for each $\varepsilon>0$ there is a $\delta>0$ such that, in the $\delta$-neighborhood of $0, X$ is the nonempty and lies in $Q_{(t-\varepsilon, t+\varepsilon)}$. If it is in $Q_{(t, t+\varepsilon)}, X$ is said to be tangential from the positive side. We make the analogous definition for the negative side.

A surface in $R^{n+k}$, which is homeomorphic to $R^{n+k-1}$ under a homeomorphism which is the identity on $R^{n}$, is called a curved horn, $\mathscr{R}_{t}$, if it is tangential to $R_{t}$ and intersects each semicircle. $C_{\beta, \alpha, x}$, at precisely one point. A curved horn tangential to $R_{t}$ on the positive (negative) side will be denoted by $\mathscr{R}_{t}^{+}\left(\mathscr{R}_{t}^{-}\right)$.

Because of the nature of the argument we shall regard as identical all curved horns which coincide in some neighborhood of 0 . Also, if two curved horns, in a neighborhood of 0 , intersect only in $R^{n}$ the region between them is defined, i.e. it is the union of the arcs on the $C_{\beta, \alpha, x}$ whose ends lie on the given horns and which lie entirely in the neighborhood. An example of the use of the convention of local identification is the validity of the statement that there is a homeomorphism taking 
$R_{0}$ to $R_{1}$ which is the identity outside a compact set. The need for such a convention will become apparent in the proof of Proposition 5.2.

Consider the trivial $R^{n+k}$-bundle over $\Delta^{m}$,

$$
\mathrm{pr}_{1}: \Delta^{m} \times R^{n+k} \rightarrow \Delta^{m} \text {. }
$$

Suppose that $K$ is a simplicial complex and that $p: K \rightarrow \Delta^{m}$ is a linear map. A map $g: K \rightarrow \Delta^{m} \times R^{n+k}$ is said to be an $f$-map if

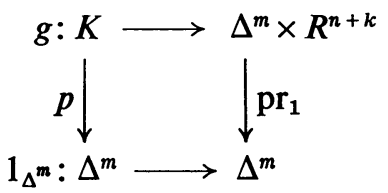

is commutative. Thus we think of $K$ as having an $f$-structure (fibered-structure) and of $g$ being an $f$-map over $1_{\Delta^{m}}$, although $p: K \rightarrow \Delta^{m}$ may not be a fiber bundle or even a fiber space.

In general, when there are $f$-structures understood, the letter " $f$ " appearing before any term will denote that the $f$-structure is to be preserved, e.g. $f$-homeomorphism, $f$-homotopy, $f$-approximation.

We extend the concepts of horn, tangentiality, etc., to $\Delta^{m} \times R^{n+k}$ by considering the inclusion of $\Delta^{m} \times R_{t}$ in $\Delta^{m} \times R^{n+k}$. A curved horn is then defined as the image of $\Delta^{m} \times R^{n+k-1}$ under a $f$-homeomorphism such that the restriction to each fiber defines a curved horn.

An $f$-map $g: K \rightarrow \Delta^{m} \times R^{n+k}$ is said to be linear if it is linear on simplices.

A set of points in $\Delta^{m} \times R^{n+k}$ is said to be in $f$-general position if every subset of $s$ points, $s \leqq m+n+k+1$, containing a maximal subset of $l$ points whose projections are in general position in $\Delta^{m}$, determines a hyperplane of dimension $(l-1)+$ $\min (n+k, s-l)$. If we take $m=0$ and $l=1$ this reduces to a standard definition of general position in $R^{n+k}$ [18].

A linear $f$-map is said to be in $f$-general position if the image of the vertices of $K$ are in $f$-general position.

4. Some basic lemmas. The first three lemmas are generalizations of standard methods.

LEMma 4.1 Suppose that $\delta^{p}$ is a simplex, with a fibered structure. If $\alpha$ denotes a disjoint vertex, then $\alpha \delta^{p}$ can be given a fibered structure extending that on $\delta^{p}$ such that there is a sequence of triangulations of $\alpha \delta^{p}, T_{j}$, with the projection onto $\delta^{p}$ simplicial and respecting the fibered structure, and such that the mesh of the $T_{j}$ 's converge to zero.

Proof. Identity $\delta^{p}$ with $\Delta^{p}$ and $\alpha$ with $e_{p+1}$, and hence $\alpha \delta^{p}$ with the geometric join of $\Delta^{p}$ and $e_{p+1}$. The line segments through points in $\Delta^{p}$ parallel to the segment $O e_{p+1}$ determine a fiber structure extending the one given on $\delta$.

The triangulations, $T_{j}$, are defined inductively as follows: 
$T_{0}$ : To the triangulation of $\partial \Delta^{p}$ add a vertex at 0 and given $\Delta^{p}$ the cone triangulation $e_{p+1} \Delta^{p}$ is also given the cone triangulation induced from the one on $\Delta^{p}$.

Suppose $T_{j-1}$ has been defined so that the projection is simplicial.

$T_{j}$ : Take a barycentric subdivision of $\Delta^{p}$, triangulated by $T_{j-1}$, ordering the barycenters in order of increasing dimension, $b \sigma_{0}, \ldots, b \sigma_{l}$. We then describe a derived subdivision of $e_{n+1} \Delta^{p}$ with respect to $T_{j-1}$ as follows:

Since the projection is simplicial the inverse image of each vertex of $T_{j}$ is a subcomplex lying in a single fiber. Take the barycentric subdivision of these complexes.

Next suppose that a derivation has been defined on $\pi^{-1}\left(\sigma_{s}\right)$ for $s<t$, where $\pi$ denotes the projection of $e_{p+1} \Delta^{p}$ to $\Delta^{p}$. Consider $\pi^{-1}\left(b \sigma_{t}\right) \subset \pi^{-1}\left(\sigma_{t}\right) . \pi^{-1}\left(b \sigma_{t}\right)$ intersects the interior of every simplex which does not lie in $\pi^{-1}\left(\partial \sigma_{t}\right)$. In the interior of every such simplex we choose the midpoint of the segment intersecting that simplex as a matrix and triangulate, in order of increasing dimension, by taking cone triangulations. This gives a triangulation of $\pi^{-1}\left(\sigma_{t}\right)$ which extends that of $\pi^{-1}\left(\partial \sigma_{t}\right)$ and is such that $\pi$ is simplicial on $\pi^{-1}\left(\sigma_{t}\right)$.

Thus we define $T_{j}$. It remains to be shown that the mesh of the $T_{j}$ 's go to zero as $j$ goes to infinity. This, however, follows as the mesh of the triangulations induced on $\Delta^{p}$ go to zero, since we take barycentric subdivisions of the complexes $\pi^{-1}\left(b \sigma_{t}\right)$ at each stage, and since the convergence property of the mesh is preserved under the identification.

Lemma 4.2. Suppose that $g: K \rightarrow \Delta^{m} \times R^{n+k}$ is an f-map, $L$ is a subcomplex of $K$ such that $\left.g\right|_{L}$ is a linear $f$-map, $\varepsilon: K \rightarrow(0, \infty)$ is a continuous function, and $P$ is a subcomplex of $\Delta^{m} \times R^{n+k}$ such that $g(K) \cap P=\varnothing$. Then there is a linear $f$-map

$$
g^{\prime}: K \rightarrow \Delta^{m} \times R^{n+k}
$$

such that $\left.g^{\prime}\right|_{L}=\left.g\right|_{L},\left\|g^{\prime}(x)-g(x)\right\|<\varepsilon(x)$, and $g^{\prime}(K) \cap P=\varnothing$.

The proof consists of taking a linear approximation to $g$ satisfying the conclusion and noting that, since $g$ is an $f$-map, the linear approximation is an $f$-map.

Lemma 4.3. Suppose that $g: K \rightarrow \Delta^{m} \times R^{n+k}$ is a linear $f$-map, $L$ is a subcomplex of $K$ such that $\left.g\right|_{L}$ is in $f$-general position, $\varepsilon: K \rightarrow(0, \infty)$ is a continuous function, and $P$ is a subcomplex of $\Delta^{m} \times R^{n+k}$ such that $g(K) \cap P=\varnothing$. Then there is a linear $f$-map in $f$-general position

$$
g^{\prime}: K \rightarrow \Delta^{m} \times R^{n+k}
$$

such that $\left.g^{\prime}\right|_{L}=\left.g\right|_{L},\left\|g^{\prime}(x)-g(x)\right\|<\varepsilon(x)$, and $g^{\prime}(K) \cap P=\varnothing$.

The proof follows from standard general position techniques, with the additional restriction that $\operatorname{pr}_{1}\left(g^{\prime}(x)\right)=\mathrm{pr}_{1}(g(x))$.

The following three lemmas are extensions of Černavskir’'s lemmas (A), (B), and the construction of his "fundamental homeomorphism". Their proofs are direct extensions of those given by Černavskiǐ and are omitted. 
Lemma 4.4. If $X$ is a subset of $\Delta^{m} \times R^{n+k}$ which lies on one side of the horn $R_{t}$ and has the property $\bar{X} \backslash X \subset \Delta^{m} \times R^{n}$, then there is a curved horn, $\mathscr{R}_{t}$, separating $X$ from $R_{t}$.

LEMmA 4.5. If the curved horns $\mathscr{R}_{t_{1}}$ and $\mathscr{R}_{t_{4}}$ intersect only at $\Delta^{m} \times R^{n}$, if $\mathscr{R}_{t_{2}}$ and $\mathscr{R}_{t_{3}}$ lie between them, and if $0 \leqq s_{1}<s_{2} \leqq 1$ are given, then, for every $\varepsilon>0$, there exists an $\varepsilon$-f-homeomorphism of $\Delta^{m} \times R^{n+k}$ over $1_{\Delta^{m}}$ which

(i) is the identity on $\Delta^{m} \times R^{n}$,

(ii) is the identity outside the e-neighborhood of $\Delta^{m} \times 0$,

(iii) is the identity on $\mathrm{cl}\left(\Delta^{m} \backslash \Delta_{s_{2}}^{m}\right) \times R^{n+k}$,

(iv) is the identity outside the region between $\mathscr{R}_{t_{1}}$ and $\mathscr{R}_{t_{4}}$, and which

(v) takes $\mathscr{R}_{t_{2}}$ to $\mathscr{R}_{t_{3}}$ over $\Delta_{s_{1}}^{m}$.

Lemma 4.6. Suppose that $s: \Delta^{m} \rightarrow[0,1]$ is a continuous function. There is a $f$-homeomorphism, $g: X_{s} \rightarrow Y_{s}$, where

$$
X_{s}=\left\{(x, y) \in \Delta^{m} \times R^{n+k} \mid y \notin \Delta_{s(x)}^{n}\right\}
$$

and

such that

$$
Y_{s}=\left\{(x, y) \in \Delta^{m} \times R^{n+k} \mid y \notin \Delta_{s(x)}^{n+1}\right\},
$$

(i) $\left.g\right|_{s^{-1}(0) \times\left(R^{n+k} \mid 0\right)}=\left.1\right|_{s^{-1}(0) \times\left(R^{n+k} \mid 0\right)}$,

(ii) $\left.g\right|_{\Delta^{m} \times\left(R^{n+k} \mid D_{4}^{n+k}\right)}=\left.1\right|_{\Delta^{m} \times\left(R^{n+k} \mid D_{4}^{n+k}\right)}$,

(iii) a sequence of points, $S$, in $Y_{s}$ converges to a point, $x$, in its complement if and only if (a) $g^{-1}(S)$ converges to a point in the complement of $X_{s}$ and (b) $g^{-1}(S)$ is tangential to the horn $R_{t}$, where $t$ is the $(n+1)$ st coordinate of $x$ and $|t| \leqq s\left(\operatorname{pr}_{1}(x)\right)$.

We shall now give a sequence of lemmas which are related to the engulfing lemmas of Stallings [16] and Zeeman [18], as extended by Černavskiı̌ [4], (cf. Bing [1], Cantrell and Lacher [3], Millett [11]). In the following, if $P$ is a complex and $Q$ is a subcomplex, then $[P \backslash Q]$ denotes the smallest subcomplex of $P$ containing $P \backslash Q$.

LEMMA 4.7. Let $P$ be a given polyhedron in $\Delta^{m} \times R^{n+k}$ containing a simplex $\sigma=\lambda \beta$, where $\lambda$ is a 1-simplex with vertices $\lambda_{0}$ and $\lambda_{1}$ contained in the same fiber, i.e. $\operatorname{pr}_{1}\left(\lambda_{0}\right)$ $=\operatorname{pr}_{1}\left(\lambda_{1}\right)$. Suppose that $\sigma \cap[P \mid \sigma]=\left[\sigma \mid \lambda_{1} \beta\right]$ and that $[P \mid \sigma]$ lies in an open set, $U$, such that $U \cap \Delta^{m} \times R^{n}=\varnothing$. If $V$ is an open set such that $\sigma \subset V, \operatorname{pr}_{1}(V) \subset \operatorname{Int} \Delta^{m}$, and $V \cap \Delta^{m} \times R^{n}=\varnothing$ there is a f-homeomorphism, $q$, of $\Delta^{m} \times R^{n+k}$ such that $q$ is the identity on $[P \mid \sigma]$ and on the complement of $V$, and $P \subset q(U)$.

Proof. By considering a model of $\lambda_{0} \beta$ lying in the appropriate Euclidean space, $R^{l} \subset R^{l+1}$, with $\lambda_{0}$ at the origin we can give $\sigma$ a fibered structure. Take $\lambda_{1}$ to be the point $(0, \ldots, 1)$ in $R^{l+1}$ and $\sigma=\lambda_{1}\left(\lambda_{0} \beta\right) . \sigma$ is then fibered by lines through the points of $\lambda_{0} \beta$ parallel to $\lambda$. Note that this fibration respects the fibration of $\sigma$ induced by that of $\Delta^{m} \times R^{n+k}$ since $\lambda$ lies in a single fiber. 
Figure 4.8 will prove useful in understanding the following construction. There exist simplices, $\lambda^{\prime}$ and $\beta^{\prime}$, such that

(i) $\lambda_{1}^{\prime} \beta^{\prime} \subset$ Int $\lambda_{1} \beta$,

(ii) $\lambda_{0}^{\prime} \subset$ Int $\lambda \beta$,

(iii) $\lambda^{\prime}$ lies in a single fiber of $\sigma$ so that the fibration of $\sigma$ determines a fibration of $\sigma^{\prime}=\lambda^{\prime} \beta^{\prime}$ having the same properties as that of $\sigma$,

(iv) $\operatorname{cl}\left(\sigma \mid \sigma^{\prime}\right) \subset U$.

Let $v_{2}$ denote the barycenter of $\lambda_{1}^{\prime} \beta^{\prime}$. On the ray determined by $\lambda_{0}^{\prime} v_{2}$ we select two points, $v_{1}$ and $v_{3}$, such that

(i) along $\lambda_{0}^{\prime} v_{2}$ we have the following order $\lambda_{0}^{\prime}, v_{1}, v_{2}, v_{3}$,

(ii) $v_{3} \lambda_{1}^{\prime} \beta^{\prime} \subset V$,

(iii) $\lambda_{0}^{\prime} v_{1}\left(\partial \lambda_{1}^{\prime} \beta^{\prime}\right) \subset U$.

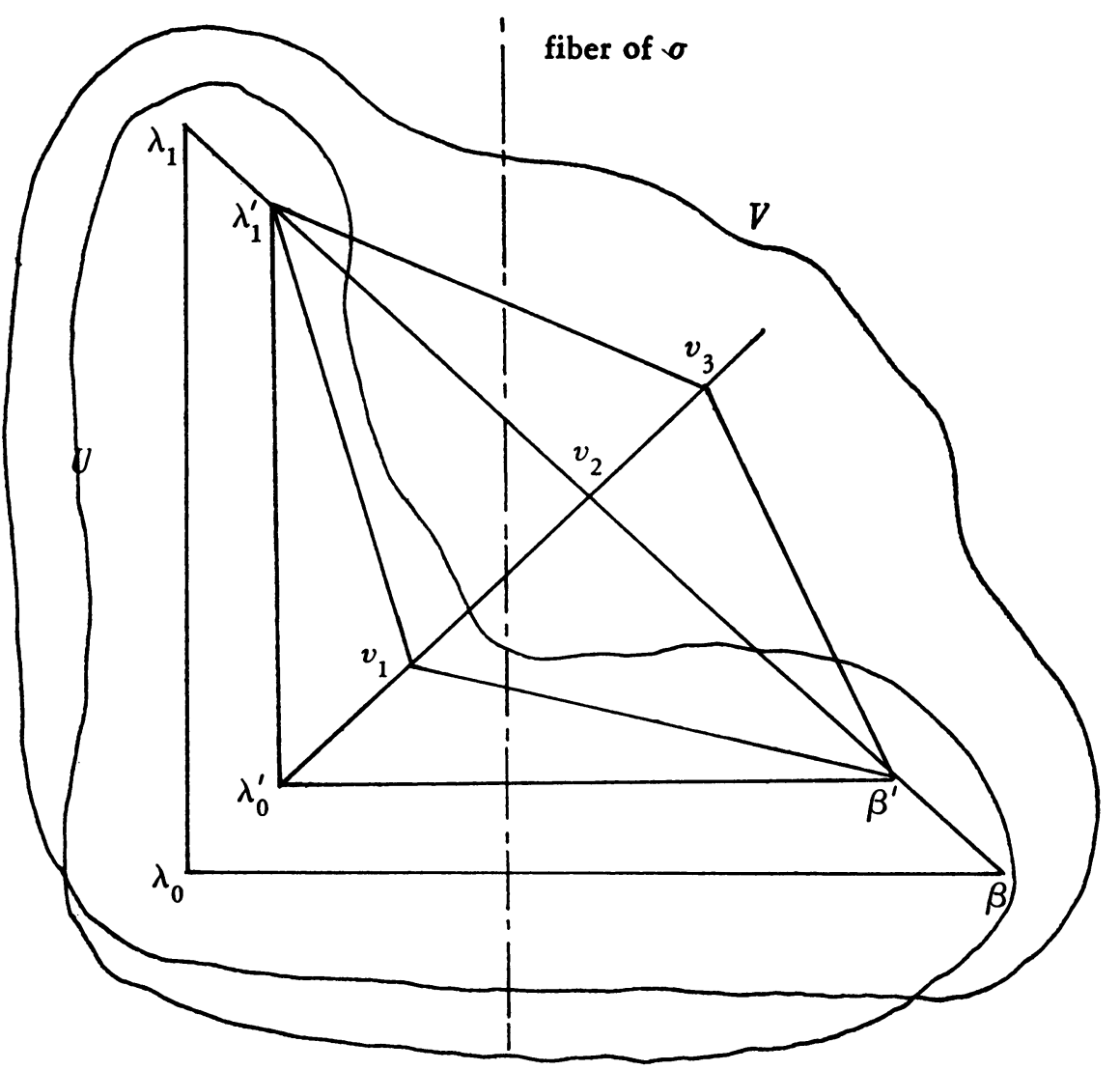

FIGURE 4.8

We note that the fibers of $\sigma^{\prime}$, extended linearly, intersect $v_{1}\left(\partial \lambda_{1}^{\prime} \beta^{\prime}\right), \lambda_{1}^{\prime} \beta^{\prime}$, and $v_{3}\left(\partial \lambda_{1}^{\prime} \beta^{\prime}\right)$ in precisely one point each, in that order. 
Select a simplex, $\delta$, in $\Delta^{m} \times R^{n+k}$ whose barycenter is at $v_{2}$ and which lies in the hyperplane orthogonal to $\sigma$. We may assume that $\delta$ is sufficiently small so that $Q=\lambda_{0}^{\prime} v_{3}(\partial \delta)\left(\partial \lambda_{1}^{\prime} \beta^{\prime}\right)$ lies entirely in $V$. Furthermore, we may assume that $v_{3}$ is sufficiently close to $v_{2}$ and that $\delta$ is sufficiently small so that $Q$ does not intersect $[P \mid \sigma]$. Finally, note that $Q$ is fibered by line segments parallel to $\lambda^{\prime}$ so that these segments intersect $v_{1}(\partial \delta)\left(\partial \lambda_{1}^{\prime} \beta^{\prime}\right),(\partial \delta)\left(\lambda_{1}^{\prime} \beta^{\prime}\right), v_{3}(\partial \delta)\left(\partial \lambda_{1}^{\prime} \beta^{\prime}\right)$ in precisely one point, in that order, and that the fibration respects that of $\Delta^{m} \times R^{n+k}$.

If, to each such line which meets the interiors, we apply the standard linear homeomorphism which leaves the endpoints fixed and which takes the point of intersection with $v_{1}(\partial \delta)\left(\partial \lambda_{1}^{\prime} \beta^{\prime}\right)$ to the point of intersection with $(\partial \delta)\left(\lambda_{1}^{\prime} \beta^{\prime}\right)$ we then define a $f$-homeomorphism of $Q$ onto itself which is the identity on the boundary and which takes $v_{1}(\partial \delta)\left(\partial \lambda_{1}^{\prime} \beta^{\prime}\right)$ to $(\partial \delta)\left(\lambda_{1}^{\prime} \beta^{\prime}\right)$. By extending this $f$-homeomorphism to the complement of $Q$ as the identity we define $q$.

Lemмa 4.9. Let $P$ be a given polyhedron in $\Delta^{m} \times R^{n+k}$ whose subpolyhedron, $Q$, is equivalent to $\sigma \times I$, with the projection onto $\sigma \times 0$ simplicial. Assume that

$$
[P \backslash Q] \cap Q \subset " \sigma \times 0 \cup(\partial \sigma) \times I "
$$

and that the projection of $Q$ to " $\sigma \times 0$ " respects the fibration of $\Delta^{m} \times R^{n+k}$. If

$$
[P \backslash Q] \cup \text { “ } \sigma \times 0 \cup(\partial \sigma) \times I " \subset U,
$$

where $U$ is an open subset of $\Delta^{m} \times R^{n+k}$ such that $U \cap \Delta^{m} \times R^{n}=\varnothing$, and $V$ is an open set containing $Q$, such that $V \cap \Delta^{m} \times R^{n}=\varnothing$ and $\operatorname{pr}_{1}(V) \subset$ Int $\Delta^{m}$, there is a $f$ homeomorphism, $q$, which is the identity outside $V$ and on $[P \mid Q] \cup$ " $\sigma \times 0 \cup(\partial \sigma) \times I$ " and has the property that $P \supset q U$.

The proof is standard, as is the proof of the following lemma.

LEMMA 4.10. Let $P$ be a given polyhedron in $\Delta^{m} \times R^{n+k}$ such that $\left[\operatorname{pr}_{1}(P)\right] \subset \operatorname{Int} \Delta^{m}$, $P \cap \Delta^{m} \times R^{n}=\varnothing$, and $P$ is finite outside of arbitrary neighborhoods of $\Delta^{m} \times 0$. Suppose that $P$ contains a countable collection of subcomplexes, $Q_{i}$, such that

(i) each $Q_{i}$ is equivalent to $\sigma_{i} \times I$, where $\sigma_{i} \times I$ has a triangulation such that projection onto $\sigma_{i} \times 0$ is simplicial and respects the fibration of $Q_{i}$ by $\Delta^{m} \times R^{n+k}$,

(ii) $\left[P \mid Q_{i}\right] \cap Q_{i} \subset " \sigma_{i} \times 0 \cup\left(\partial \sigma_{i}\right) \times I "$,

(iii) each $Q_{i}$ is contained in an open set, $V_{i}$, such that $V_{i} \cap \Delta^{m} \times R^{n}=0$, the diameters of the $V_{i}$ converge to zero, and $\mathrm{pr}_{1}\left(\mathrm{cl}\left(\bigcup_{i} V_{i}\right)\right) \subset \operatorname{Int} \Delta^{m}$,

(iv) $Q_{i} \cap Q_{j} \subset$ “ $\sigma_{i} \times 0 \cup \sigma_{j} \times 0 \cup\left(\partial \sigma_{i}\right) \times I \cup\left(\partial \sigma_{j}\right) \times I ", \quad i \neq j$,

(v) $\left[P \mid \cup Q_{i}\right] \subset U, U$ an open set in $\Delta^{m} \times R^{n+k}$ such that $U \cap \Delta^{m} \times R^{n}=\varnothing$.

For any given $\varepsilon>0$ there is a f-homeomorphism, $q$, of $\Delta^{m} \times R^{n+k}$ which is the identity on $\Delta^{m} \times R^{n}, \Delta^{m} \times e_{n+1},\left[P \mid \bigcup_{i} Q_{i}\right]$, the complement of $\bigcup_{i} V_{i}$, and the complement of an $\varepsilon$-neighborhood of $\Delta^{m} \times 0$, and which has the property that $[P \mid R] \subset q U$, where $R$ is some finite subset of the $Q_{i}$. 
Lemma 4.11. Suppose that $k \geqq 4$ and $m+n \leqq 2(k-3)$. Let $P$ be a polyhedron in $\Delta_{t}^{m} \times R^{n+k}, 0 \leqq t<1$, such that $P \cap \Delta^{m} \times R^{n}=\varnothing, P$ is finite outside arbitrary neighborhoods of $\Delta^{m} \times 0, \operatorname{dim} P \leqq m+n-k+4$ and $\operatorname{dim} P \cap(x) \times R^{n+k} \leqq n-k+3, x \in \Delta^{m}$. Let $U$ be a region which is f-homeomorphic to $\Delta^{m} \times R_{n}^{+}$via an $f$-hameomorphism which is the identity on $\Delta^{m} \times R^{n}$. Finally, let $Q$ be a polyhedron of dimension $m+n+1$ which is contained in $U$ and is finite outside arbitrary neighborhoods of $\Delta^{m} \times 0$.

For any given $\varepsilon>0$ there is an $s, t<s<1$, and an $\varepsilon-f$-homeomorphism

$$
q: \Delta^{m} \times R^{n+k} \rightarrow \Delta^{m} \times R^{n+k}
$$

which is the identity on $\Delta^{m} \times R^{n}, \Delta^{m} \times e_{n+1}, Q,\left(\Delta^{m} \backslash \Delta_{s}^{m}\right) \times R^{n+k}, Q$ and outside the $\varepsilon$-neighborhood of $\Delta^{m} \times 0$, with the property that

$$
q(\text { Int } U) \supset[P \backslash R] \cup Q
$$

where $R$ is some finite subcomplex of $P$.

Proof. The proof is by induction on the dimension of $P$. The induction begins with $\operatorname{dim} P=-1$, i.e. $P=\varnothing$. Suppose, then, that the lemma has been proven for polyhedra of dimension smaller than $\operatorname{dim} P$.

Thus we may assume that we have constructed an $f$-homeomorphism

$$
q^{\prime}: \Delta^{m} \times R^{n+k} \rightarrow \Delta^{m} \times R^{n+k}
$$

such that the conclusion holds for the ( $p-1)$-skeleton of $P, s^{\prime}, \varepsilon / 2$ and $R$.

Subdivide $P$ so that every simplex which intersects $Q$ lies in $q^{\prime}($ Int $U)$ and index the $p$-simplexes which neither intersect $R$ nor lie in $q^{\prime}($ Int $U)$ by $\sigma_{0}, \sigma_{1}, \ldots$ Note that the diameters of the $\sigma_{i}$ converge to zero.

Let $\Delta^{p+1}$ denote the standard $(p+1)$-simplex written as $\alpha \delta, \delta$ a fixed $p$-face of $\Delta^{p+1}$ and $\alpha$ a fixed vertex. For each $i$ there is a linear homeomorphism of $\delta$ onto $\sigma_{i}$ which, then, gives rise to the notion of an $f$-map, for each $i$, of $\Delta^{p+1}$ into $\Delta^{m} \times R^{n+k}$ by requiring that the fibers of $\alpha \delta$ respect the structure already given on $\delta$ via the linear homeomorphism.

The map of $\partial \sigma$ to $\partial \sigma_{i}$ is an $f$-map of $\partial \delta$ into $q^{\prime}($ Int $U)$. We can then extend to an $f$-map of $\alpha \partial \delta$ such that the diameters of these images converge to zero. Thus we have defined a sequence of $f$-maps

$$
\theta_{i}: \partial \Delta^{p+1} \rightarrow \Delta^{m} \times R^{n+k}
$$

such that $\theta_{i}(\delta)=\sigma_{i}$ and $\theta_{i}(\alpha \partial \delta) \subset q^{\prime}($ Int $U)$ which we wish to extend to $\Delta^{p+1}$ such that the images do not intersect $\Delta^{m} \times R^{n}$ and such that the diameters of the images converge to zero. This is possible since $p \leqq m+n-k+4$ and $m+n \leqq 2(k-3)$, so that $p \leqq k-2$.

We wish to take a linear $f$-approximation of each $\theta_{i}$, relative to $\left.\theta_{i}\right|_{\delta}$, such that with respect to the subdivision, the projection of $\Delta^{p+1}$ onto $\delta$ is simplicial. This is possible by Lemmas 4.1 and 4.2, using the triangulations of 4.1 in 4.2. By Lemma 
4.3 and $\theta_{i}$ are brought into $f$-general position with respect to $P$ and $Q$ without moving those vertices of $\theta_{i}(\Delta)$ already in $P$. Furthermore the $\theta_{i}$ 's have the property that the diameters of the images converge to zero and the images do not meet $\Delta^{m} \times R^{n}$. We may also require, without loss of generality, that the images lie in $\Delta_{s_{1}}^{m} \times R^{n+k}, s^{\prime} \leqq s_{1}<1$.

We shall now consider the singular set of the $\theta_{i}$, i.e. the intersections of the $\theta_{i}\left(\Delta^{p+1}\right)$ with themselves and $P$ and $Q$. From the definition of $f$-general position we have

$$
\begin{aligned}
\operatorname{dim}\left(\theta_{i}\left(\Delta^{p+1}\right) \cap \theta_{j}\left(\Delta^{p+1}\right)\right) & \leqq\left(\operatorname{dim}\left(P \cap(x) \times R^{n+k}\right)+1\right)+(\operatorname{dim} P+1)-(n+k) \\
& \leqq(n-k+3+1)+(m+n-k+4+1)-(n+k) \\
& =m+n-3 k+9 \leqq 3-k, \\
\operatorname{dim}\left(\theta_{i}\left(\Delta^{p+1}\right) \cap P\right) & \leqq(n-k+3+1)+(m+n-k+4)-(n+k) \\
& =m+n-3 k+8 \leqq 2-k, \\
\operatorname{dim}\left(\theta_{i}\left(\Delta^{p+1}\right) \cap Q\right) & \leqq(n-k+3+1)+(m+n+1)-(n+k) \\
& =m+n-2 k+5 \leqq-1 .
\end{aligned}
$$

Thus, if $k \geqq 4$, the singular set is empty. Hence we may apply Lemma 4.10 to the images of the $\theta_{i}(\Delta)$, since the projections onto $\theta_{i}(\delta)$ are simplicial, thereby defining the " $Q_{j}$ " as those inverse images which are not entirely in $q^{\prime}($ Int $U)$. Note that we may assume the triangulation is sufficiently fine so that the " $Q_{j}$ " have the required structure.

The following two lemmas, which are generalizations of the Alexander isotopy, were used in $\S 2$ to conclude the proof of Theorem 2.1 .

LEMMA 4.12. Suppose that $G_{2}: \Delta^{m} \times R^{n+k} \rightarrow \Delta^{m} \times R^{n+k}$ is an f-homeomorphism and $t_{1}<1<t_{2}$ are such that $G_{2}$ is the identity on

$$
\Delta^{m} \backslash \Delta_{t_{1}}^{m} \times R^{n+k} \cup \Delta^{m} \times R^{n} \cup \Delta^{m} \times D_{t_{2}}^{n+k}
$$

then $G_{2}$ is f-isotopic to the identity through such f-homeomorphisms.

The proof is the same as for Alexander isotopy.

LEMMA 4.13. Suppose that $G_{1}: \Delta^{m} \times R^{n+k} \rightarrow \Delta^{m} \times R^{n+k}$ is an f-homeomorphism which

(i) is the identity on $\partial \Delta^{m} \times R^{n+k}$,

(ii) is the identity on $\Delta^{m} \times\left(R^{n+k} \backslash D_{4}^{n+k}\right)$,

(iii) is the identity on $\Delta^{m} \times R^{n}$,

(iv) is the identity on $\Delta^{m} \times\left(e_{n+1}\right)$.

Then $G_{1}$ is f-isotopic, through f-homeomorphisms satisfying (i), (iii), (iv) to one which is the identity on $\Delta^{m} \times R^{n+1}$.

Proof. $G_{1}$ is $f$-isotopic to the identity through $f$-homeomorphisms satisfying (i), (ii) and (iii). However, we lose (iv). In regaining (iv) we lose (ii), as noted in the conclusion. 
We may assume, without loss of generality, that the track of $\Delta^{m} \times\left(e_{n+1}\right)$ is in $\Delta^{m} \times R^{k}$ since we may translate in the complement of $\Delta^{m} \times R^{n}$.

Let $R_{+}^{1} O(k)$ denote the group consisting of orthogonal matrices multiplied by a positive scalar and let $p(w)=w\left(e_{1}\right), w \in R_{+}^{1} O(k)$. Then

$$
O(k-1) \rightarrow R_{+}^{1} O_{k} \underset{\mathrm{p}}{\longrightarrow} R^{k} \backslash O
$$

is a fibration and has a regular lifting function, $\lambda$. Since the track of the $f$-isotopy remains in $\Delta^{m} \times R^{k}$ we may use the track and the regular lifting function to describe a family in $R_{+}^{1} O(k)$ which is used to regain (iv). Since the track is constant over $\partial \Delta^{m}$ and the lifting function is regular, (i) is satisfied.

5. The main propositions. The two propositions contained in this section constitute a major portion of the theory developed to prove Theorem 2.1. The first of these, for which a proof is included, provides the means to prove the second. The proof of the second proposition is a generalization of an argument given by Černavskir [4]. Since his argument is very long and since the author has not significantly simplified it we shall only give an outline of the proof to the extent needed to indicate the modifications which must be made in Černavskiř's proof.

Proposition 5.1. Suppose $k \geqq 4 m+n \leqq 2 k-6$ and $\tau \in R^{1} \mid 0$. Let $s<1$ and $G$ be an f-homeomorphism such that

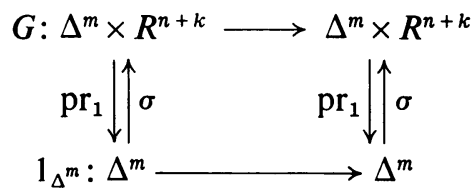

commutes, where $\sigma(x)=\left(x, e_{n+1}\right)$.

(ii) $G$ is the identity on $\Delta^{m} \times R^{n}$.

(iii) $G$ is the identity on $\left(\Delta^{m} \backslash \Delta_{s}^{m}\right) \times R^{n+1}$.

Then there is an $r, s<r<1$, and an $f$-homeomorphism

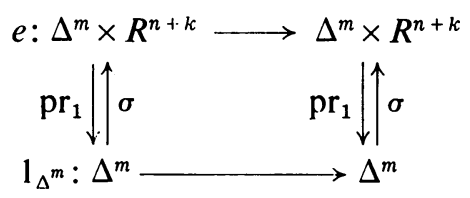

such that

(i) the diagram commutes, where $\sigma(x)=\left(x, e_{n+1}\right)$,

(ii) $e$ is the identity on $\Delta^{m} \times\left(R^{n} \cup\left(R^{n+k} \backslash D_{1 / 2}^{n+k}\right)\right)$,

(iii) $e$ is the identity on $\left(\Delta^{m} \backslash \Delta_{s_{1}}^{m}\right) \times R^{n+k}$,

(iv) there is a $t>0$ such that

$$
e\left(G Q_{\tau}^{\operatorname{sgn} \tau}\right) \supset \Delta^{m} \times \Delta_{t}^{n+1} \operatorname{sgn} \tau .
$$


Furthermore, if $G\left(Q_{\tau}^{\operatorname{sgn} \tau}\right)$ lies on one side of a horn which contains $\Delta^{m} \times \Delta_{t}^{n+1} \operatorname{sgn} \tau$, $t>0$, then e can be taken to be the identity on the other side of the horn.

Proof. We may, without loss of generality, assume $\tau>0$ since the proof for $\tau<0$ is similar. Let $B_{1}=\Delta^{m} \times \Delta_{+}^{n+1} \subset \Delta^{m} \times R^{n+1}$ and $\mathscr{B}_{1}=G\left(B_{1}\right)$. Let $\Delta$ denote the $(n+2)$-simplex, defined as $v \Delta_{+}^{n+1}$, and $D=\Delta^{m} \times \Delta$, with subsets $B_{2}=\Delta^{m} \times \Delta_{+}^{n+1}$, $\mathscr{B}_{2}=\Delta^{m} \times\left(\Delta^{n} * v\right)$ and $C=\Delta^{m} \times\left(e_{n+1} * v\right)$.

There is an $f$-map $\phi: D \rightarrow \Delta^{m} \times R^{n+k}$ such that

(i) $B_{2}$ is mapped linearly onto $B_{1}$ via the identification,

(ii) if $\alpha$ denotes the linear projection of $D$ onto $B_{2}$, then $\left.\phi\right|_{\mathscr{D}_{2}}=\left.G \phi \alpha\right|_{\mathscr{B}_{2}}$,

(iii) $\phi(C) \subset \Delta^{m} \times\left(e^{n+1}\right)$,

(iv) $\phi\left(D \backslash\left(\Delta^{m} \times \Delta^{n}\right)\right) \subset \Delta^{m} \times\left(R^{n+k} \backslash R^{n}\right)$,

(v) $\phi\left|\operatorname{cl}\left(\Delta^{m} \mid \Delta_{s}^{m}\right) \times \Delta=\phi \alpha\right| \operatorname{cl}\left(\Delta^{m} \backslash \Delta_{s}^{m}\right) \times \Delta$.

By Lemmas 4.1, 4.2 and 4.3 there is a linear general position $f$-map, also denoted by $\phi$,

$$
\phi: D \backslash\left(\Delta^{m} \times \Delta^{n}\right) \rightarrow \Delta^{m} \times\left(R^{n+k} \mid R^{n}\right)
$$

and $s_{1}, s<s_{1}<1$ such that

(i) $B_{2} \mid\left(\Delta^{m} \times \Delta^{n}\right)$ is mapped linearly into $B_{1}$ via the identity,

(ii) $\phi\left(\mathscr{B}_{2}\right) \subset$ Int $G\left(Q_{\tau}^{+}\right)$,

(iii) $\phi(C) \subset$ Int $G\left(Q_{\tau}^{+}\right)$,

(iv) $\phi\left(\left(\Delta^{m} \backslash \Delta_{s_{1}}^{m}\right) \times\left(\Delta \backslash \Delta^{n}\right)\right) \subset G\left(Q_{\tau}^{+}\right)$,

(v) with respect to the triangulation of $D \backslash \Delta^{m} \times R^{n}, \alpha$ is simplicial,

(vi) $\phi$ extends to a continuous map on $D$ (agreeing with the previous map on $\left.\Delta^{m} \times \Delta^{n}\right)$.

Note that, in general, $\mathscr{B}_{1}$ will not be equal to $\phi\left(\mathscr{B}_{2}\right)$.

Following R. H. Bing [1], we define the shadow for $\phi$ by taking $S$ to be those points which are identified under $\phi$ and such that, for any such pair, at least one lies in the interior of a simplex of dimension less than $m+n+2$. A polyhedron, in $\Delta_{s_{1}}^{m} \times \Delta$ is called a shadow for $\phi$ if

(i) $\operatorname{dim} P \leqq m+n-k+4$,

(ii) $P=\alpha^{-1}(\alpha(P))$,

(iii) if $x, y \in \Delta_{s_{1}}^{m} \times \Delta, \phi(x)=\phi(y), \quad x \in P$ if and only if $y \in P$.

The existence of a shadow is proved using Lemma 4.3 , i.e. $f$-general position, and Bing's shadow building theorem [1].

Applying Lemma 4.11 we engulf the shadow, $P$, save for some finite subcomplex, $R_{1}$. Subdivide so that $P$ is a subcomplex, as in Lemma 4.1 , and such that $\alpha$ is simplicial. By induction, using Lemma 4.10, we engulf the $m+n+1$ skeleton, except for a finite subcomplex, $R_{2}$, which depends on $R_{1}$. Finally we engulf $B_{2}$, except for a finite subcomplex, by applying Lemma 4.7 to this situation in the same way that it was used to prove Lemma 4.10, through 4.8 and 4.9. Note that in each application we cover an $m+n+2$ simplex, but, when passing to the one below it, its interior need not remain covered since $P$ does not contain 
all of the singularities of $\phi$. We also note that an infinite process is involved. This is accomplished as in Lemmas 4.9 and 4.10.

Since we have engulfed $B_{2}$, save for a finite subcomplex, the first part of the theorem follows. The remainder is proved by observing that if $G\left(Q_{\tau}^{+}\right)$lies on one side of a horn which contains $\Delta^{m} \times \Delta_{t^{+}}^{n+1}$, then the image of $\phi$ and resulting engulfing may be taken to lie on the desired side of the horn. Thus, the $f$-homeomorphism which is the result of the engulfing process is the identity on the other side of the horn.

Proposition 5.2. Suppose that

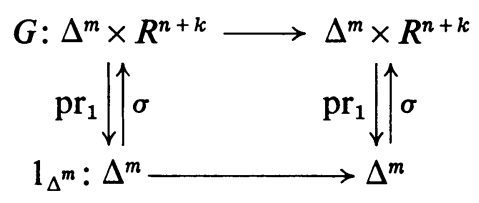

is a bundle map such that

(i) the diagram commutes, where $\sigma(x)=\left(x, e_{n+1}\right)$,

(ii) $G$ is the identity on $\Delta^{m} \times R^{n}$,

(iii) there is an $s<1$ such that $G$ is the identity on $\left(\Delta^{m} \backslash \Delta_{s}^{m}\right) \times R^{n+1}$.

If $k \geqq 4$ and $m+n \leqq 2 k-6$, then $G=G_{1} G_{2} G_{3}$, where each $G_{i}$ has the properties of $G$ and, in addition, there are numbers, $t_{1}<1<t_{2}$, such that

(i) $G_{1}$ is the identity on

$$
\left(\Delta^{m} \backslash \Delta_{t_{1}}^{m}\right) \times R^{n+k} \cup \Delta^{m} \times R^{n} \cup \Delta^{m} \times\left(R^{n+k} \mid D_{4}^{n+k}\right) .
$$

(ii) $G_{2}$ is the identity on

$$
\left(\Delta^{m} \mid \Delta_{t_{1}}^{m}\right) \times R^{n+k} \cup \Delta^{m} \times R^{n} \cup \Delta^{m} \times D_{t_{2}}^{n+k} .
$$

(iii) $G_{3}$ coincides with $G$ over $\Delta^{m} \backslash \Delta_{t_{1}}^{m}$ and is the identity on $\Delta^{m} \times R^{n+1}$.

Proof. We shall employ the notation and terminology used by Černavskiř [4] in proving his main theorem, as extended in this work. We shall, also, assume familiarity with the proof of this theorem so that it will only be necessary to indicate where, and how, modifications are to be made in his argument.

Following Černavskir we first construct the $f$-homeomorphism, $G_{1}$, satisfying condition (i) and, furthermore, such that $G^{\prime}=G_{1}^{-1} G$ is the identity on $\Delta^{m} \times D_{2}^{n+1}$. The construction of $G_{1}$ follows from Proposition 5.1 and Lemmas 4.4, 4.5, and 4.6 using the associated techniques of Černavskin. It is possible to make a slight simplification in his argument so that it is only necessary to use Proposition 5.1 twice (cf. [3] or [11]). One then uses Lemmas 4.4 and 4.5 to modify the $f$-homeomorphism so that it preserves the tangentiality of the horns, $R_{t},|t| \leqq 2$. Lemma 4.6 is used to define $G_{1}$ as in [4].

We next construct a $f$-homeomorphism, $G_{3}$, such that $G_{3}=G_{2}^{-1} G^{\prime}$, i.e. we construct $G_{3}$, take $G_{2}=G^{\prime} G_{3}^{-1}$, and observe that $G_{2}$ has the desired properties. As with 
the construction of $G_{1}$ we parallel the related construction given in [4]. To construct $G_{3}$ one first constructs a sequence of concentric "balls", $B_{i}$, and $f$-homeomorphisms, $H_{i}, i=-1,0,1, \ldots$ Each "ball" is determined by a function $\gamma_{i}: \Delta^{m} \rightarrow\left[t_{2}, \infty\right]$, $i \geqq 0$, such that $\gamma_{i}^{-1}(\infty) \supset\left(\Delta^{m} \backslash \Delta_{t_{1}}^{m}\right)$ as follows:

$$
B_{i}=\left\{(x, y) \in \Delta^{m} \times R^{n+k} \mid\|y\| \leqq \gamma_{i}(x)\right\} .
$$

The $f$-homeomorphisms and "balls" shall satisfy the following conditions:

(1) $B_{i} \subset B_{i+1}$,

(2) $\bigcup_{i \geq 1} B_{l}=\Delta^{m} \times R^{n+k}$,

(3) $H_{i}\left(B_{i}\right) \subset$ Int $B_{i+1}$,

(4) $H_{i}\left(B_{i}\right) \supset B_{i-1}$,

(5) $\left.H_{i}\right|_{B_{i-1}}=\left.H_{i-1}\right|_{B_{i-1}}$,

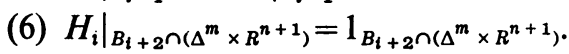

They are constructed by induction, taking $H_{-1}=G$ and $\gamma_{-1}(x)=t_{2}$ (for an appropriate $t_{2}$ ), as in [4]. The only modification in the argument is that one works with continuous functions on $\Delta^{m}$ instead of constants as in [4]. $G_{3}$ is defined by

$$
\left.G_{3}\right|_{B_{i}}=\left.H_{i}\right|_{B_{i}}
$$

\section{REFERENCES}

1. R. H. Bing, Radial engulfing, Conference on Topology of Manifolds, Michigan State Univ. (mimeographed, 1967).

2. W. Browder, Open and closed disk bundles, Ann. of Math. (2) 83 (1966), 218-230.

3. J. C. Cantrell and R. C. Lacher, Some conditions for manifolds to be locally flat (mimeographed, 1967).

4. A. V. Cernavskiĭ, Homeomorphisms of Euclidean space and topological embeddings of polyhedra in Euclidean space, Mat. Sb. 63 (1965), 581-613.

5. A. Haefliger and C. T. C. Wall, Piecewise linear bundles in the stable range, Topology 4 (1965), 109-214.

6. M. W. Hirsch, On non-linear cell bundles, Ann. of Math. (3) 84 (1966), 373-385.

7. D. Husemoller, Fiber bundles, McGraw-Hill, New York, 1966.

8. R. Kirby and L. Siebenmann, On the triangulation of manifolds and the Hauptvermutung, Bull. Amer. Math. Soc. 75 (1969), 742-749.

9. J. M. Kister, Microbundles are fiber bundles, Ann. of. Math. (2) 80 (1964), 190-199.

10. R. Lashof and M. Rothenberg, Triangulation of manifolds. I, II, Bull. Amer. Math. Soc. 75 (1969) 750-752.

11. K. C. Millett, The theory of euclidean bundle pairs: Homotopy normal bundles and nonzero sections, Doctoral thesis, Univ. of Wisconsin, Madison, 1967.

12. - Homotopy normal bundles for locally flat immersions and embeddings of topological manifolds, Illinois J. Math. 13 (1969), 564-572.

13. - Normal structures for locally flat embeddings, Proc. Amer. Math. Soc. 20 (1969), 580-584.

14. J. Milnor, Construction of universal bundles. II, Ann. of Math. (3) 63 (1956), 430-436.

15. C. P. Rourke and B. J. Sanderson, On embedding without a normal microbundle, Invent. Math. 3 (1967), 293-299. 
16. J. Stallings, The piecewise linear structure of Euclidean space, Proc. Cambridge Philos. Soc. 58 (1962), 481-488.

17. J. H. C. Whitehead, Combinatorial homotopy. I, Bull. Amer. Math. Soc. 55 (1949), 213-243.

18. E. C. Zeeman, Seminar on combinatorial topology. I, II (mimeographed notes), Inst. Hautes Études Sci., Paris, 1963.

MassachusetTs Institute of Technology,

Cambridge, Massachusetts 